\title{
Producción de pigmentos por Monascus spp. en medio sólido empleando residuos agroindustriales
}

\author{
Pigments production by Monascus spp. in solid medium \\ using agroindustrial waste

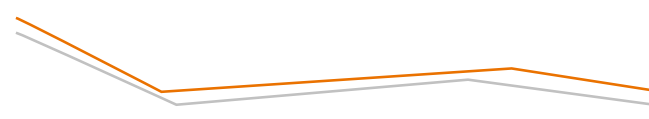 \\ María Elena Velázquez Arellano'*, Juan Roberto Benavente Valdés', \\ Jesús Antonio Morlett Chávez', Cristóbal Noé Aguilar González' \\ Velázquez Arellano, M. E., Benavente Valdés, J. R. Morlett Chávez, J. A., Aguilar González, C. N. \\ Producción de pigmentos por Monascus spp. en medio sólido empleando residuos agroindustriales. \\ Investigación y Ciencia de la Universidad Autónoma de Aguascalientes. Número 69: 89-95, \\ septiembre-diciembre 2016 .
}

\section{RESUMEN}

En esta revisión se describen los principales factores que afectan la producción de pigmentos por Monascus en medio sólido, así como los diferentes residuos utilizados y aquellos potenciales remanentes regionales en México que pueden ser aprovechados en la producción de pigmentos. Los colorantes producidos por el hongo Monascus han mostrado potencial aplicación en las industrias alimentaria, cosmética, farmacéutica y textil. La producción de pigmentos por Monascus se ha llevado a cabo principalmente en cultivos en estado sólido, para lo cual el uso de residuos agroindustriales como fuente de sustrato es una alternativa real que incrementa la factibilidad económica del proceso, aunado a ser una alternativa de bajo impacto ambiental. Los factores de temperatura, $\mathrm{pH}$, humedad relativa y tamaño de partícula se identificaron como los factores de mayor influencia en la producción de pigmentos. Los residuos regionales de maíz, arroz y Aloe vera son los sustratos con mayor rendimiento.

ABSTRACT

In this review the main factors affecting pigment production by Monascus in solid medium are

Palabras clave: colorantes naturales, hongo filamentoso, fermentación sólida, tecnologías sustentables.

Keywords: natural dyes, filamentous fungus, solid-state fermentation, sustainable technologies.

\section{Recibido: 3 de noviembre de 2015, aceptado: 19 de agosto de 2016}

Departamento de Investigación en Alimentos, Universidad Autónoma de Coahuila.

* Autor para correspondencia: mvelazquez@uadec.edu.mx described, as well as the different agro-industrial waste used and those potential regional wastes in Mexico that can be exploited in the production of pigments. The pigments produced by the fungus Monascus have shown potential application in food, textile, cosmetics and pharmaceutical industries. Pigment production by Monascus has been carried out mainly in solid-state fermentation, for which the use of agro-industrial waste as a source of substrate is a real alternative that increases the economic feasibility of the process with low-impact environmental. The factors of temperature, $\mathrm{pH}$, humidity and particle size were identified as the most influential factors in pigment production and the regional agro-industrial waste of corn, rice and Aloe vera the substrates with higher yield.

\section{INTRODUCCIÓN}

Los pigmentos se definen como sustancias químicas que imparten color a otros materiales por el efecto óptico de la refracción de la luz del sol (Wani et al., 2004). Los colorantes naturales fueron una parte esencial dentro de diversas industrias en el siglo XIX (Bechtold et al., 2006); sin embargo, debido a la gran demanda de estas moléculas a nivel industrial, los colorantes de origen natural fueron reemplazados por los sintetizados vía química. Los colorantes sintéticos han suplido casi por completo a los naturales, debido a la amplia gama de colores disponibles y a la alta eficiencia de producción a bajo costo, principalmente. Sin embargo, ha sido bien documentado que los colorantes químicamente sintetizados han presentado efectos negativos en la salud humana, tales como alergias, hiperactividad en infantes y en algunos casos se han asociado con cáncer (Hsu y Pan, 2012). Por tanto, hoy día diversas 
empresas se han enfocado en la búsqueda de alternativas a estos colorantes, siendo los de origen natural una potencial fuente de sustitución en la coloración de una amplia gama de productos.

Las fuentes originales de tintes naturales han sido las plantas y tejidos de insectos; sin embargo, también es factible producir pigmentos a través de microorganismos tales como bacterias, levaduras, hongos y microalgas (Celestino et al., 2014; Zang et al., 2014; Morales Oyervides et al., 2015; Streit et al., 2015; Benavente Valdés et al., 2016; Dursun y Dalgiç, 2016). Dentro de la producción microbiana de pigmentos, el hongo Monascus spp. es uno de los microorganismos fúngicos ampliamente reconocidos por su capacidad de producción de los mismos (Babitha et al., 2007; Velmurugan et al., 2011; Yang et al., 2015).

El género Monascus pertenece a la clase Ascomycetos y a la familia Monascaceae. Este hongo es fuente de varios metabolitos secundarios, tales como monacolina, ácido gama-aminobutírico y pigmentos policétidos del tipo azafilonas (Wang et al., 2015). Dentro de los pigmentos producidos por Monascus se han reportado monascina y ankaflavina, correspondientes a colores amarillos; rubropunctatina y monascurubrina, correspondientes a tonalidades naranjas y rubropuctamina y monascurubramina, correspondientes a la gama de los rojos (Carvalho et al., 2003). Dentro de estos colorantes con perspectiva industrial, los rojos han tenido mayor demanda, especialmente en la industria de los alimentos ( $\mathrm{Li}$ et al., 2014). Por otro lado, los pigmentos amarillos y naranjas han sido ampliamente estudiados debido a sus propiedades biológicas (Cheng et al., 2012; Nam et al., 2014).

La obtención de pigmentos de Monascus es llevada a cabo principalmente en cultivo en estado sólido, ya que el proceso tiene las siguientes ventajas: ॥) proceso simple de implementar, ॥) menor inversión de capital, ıI) bajos niveles de inhibición del producto final y represión catabólica, iv) menor cantidad de residuos líquidos generados, v) mayor rendimiento en general (Babitha et al., 2007; Lian et al., 2007; Pongrawee y Lumyong, 2011; Velmurugan et al., 2011; General et al., 2014).

Desde una perspectiva industrial existe la necesidad de desarrollar sistemas de bajo costo para la producción de colorantes y hacer factible su producción masiva, ya que la principal limitante son los altos costos asociados al sustrato. Una alternativa interesante a escala industrial es el empleo de residuos agroindustriales para la biosíntesis de pigmentos fúngicos, así como la exploración de residuos no convencionales generados regionalmente, con lo cual se impulsará el desarrollo de empresas biotecnológicas en el país.

Con base en lo anterior, el objetivo de esta contribución es presentar un panorama general de la información reportada referente a los principales factores involucrados en la producción de pigmentos por Monascus en cultivos en estado sólido; además de presentar las áreas de oportunidad con base en los subproductos agrícolas generados en el norte de México y pueden ser empleados en la producción de tintes.

\section{Fermentación en estado sólido}

La fermentación en medio sólido (FMS) se define como el crecimiento de microorganismos sobre un sustrato sólido húmedo, donde los niveles de humedad son suficientes para mantener el crecimiento microbiano y llevar a cabo su metabolismo; sin embargo, no hay agua libre en movimiento (Rahardjo et al., 2006). En estos procesos, los sustratos no solo suministran los nutrientes al microorganismo, sino que sirven como medio para la inmovilización de las células (Pandey, S. et al., 2007). Por tanto, en FMS se presenta un hábitat más adecuado para el crecimiento de los hongos, lo que permite altas productividades del metabolito de interés (Pandey, A. et al., 2000; Pandey, S. et al., 2007).

En países orientales como China, el cultivo de Monascus en sistemas sólidos con arroz como sustrato es utilizado para la elaboración de Anka (Figura 1), un arroz fermentado altamente demandado (Dufossé et al., 2005). Sin embargo, se han utilizado diversas materias primas como sustrato para fermentación con cepas de Monascus spp.

Las industrias alimentarias, granjas de giro agrícola y forestal generan grandes volúmenes de desechos, los cuales pueden ser utilizados como materia prima para los procesos de FMS. Algunos ejemplos de ellos son residuos olote de maíz, bagazo de yuca, bagazo de caña, salvado de trigo, salvado de arroz, vaina de algarroba, residuos de la industria cervecera, entre otros (Rodríguez Couto, 2008). Previo a la utilización de material en FMS es de gran importancia determinar las características microbiológicas y fisicoquímicas de los materiales a ser empleados como sustratos. Dentro de estos 


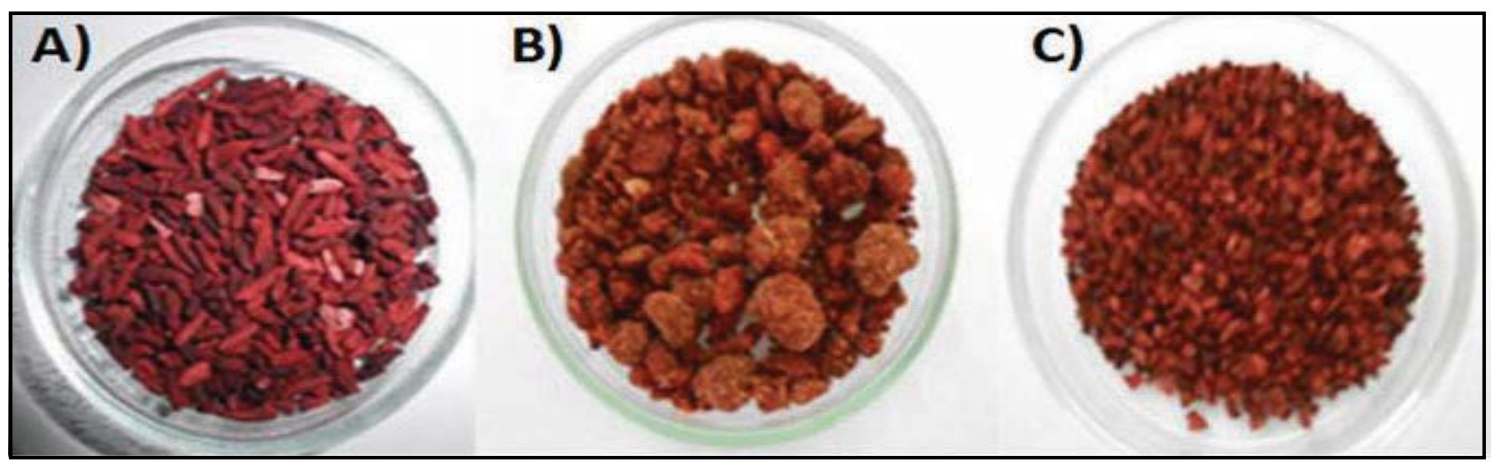

Figura 1. Monascus purpureus HD001 fermentado después de 14 días en A) arroz, B) residuos de tofu y C) gadung (tubérculo) (Priatni et al., 2014).

existen dos parámetros fisicoquímicos de suma importancia para la selección de un sustrato, índice de absorción de agua (IAA) y el punto crítico de humedad $(\mathrm{PCH})$, que representa la cantidad de agua ligada al soporte que no puede ser empleada por el microorganismo y se encuentra fuertemente relacionado con la actividad de agua (aw). Los valores superiores a $40 \%$ de $\mathrm{PCH}$ indican que el agua no se encuentra ligada al soporte, sino en solución y, por tanto, afecta el crecimiento fúngico, en cultivos sólidos se requiere que los $\mathrm{PCH}$ sean bajos para poder modificar el valor de la humedad en función del contenido del medio absorbido (Robledo et al., 2008).

Diversos autores han reportado el uso de residuos agroindustriales para la producción de colorantes de Monascus en FMS, los cuales se describen en la Tabla 1.

Dentro del proceso de biosíntesis de tintes en FMS, algunos autores han reportado que son varios los factores que afectan el rendimiento de producción, dentro de los cuales han mencionado la temperatura, $\mathrm{pH}$, humedad relativa, tamaño de partícula y flujo de aireación (Han y Mudgett, 1992; Carvalho et al., 2003; Dufossé et al., 2005; Babitha et al., 2007; Velmurugan et al., 2011).

\section{Factores físicos que influyen en la producción de pigmentos en medio sólido}

Temperatura y $\mathbf{p H}$. La temperatura es un factor determinante para el crecimiento y metabolismo de las células. La mayoría de los cultivos fúngicos en FMS han sido llevados a cabo en rangos de temperatura de 10 a $35^{\circ} \mathrm{C}$, con un óptimo entre 20 y $30^{\circ} \mathrm{C}$. Diversos estudios con Monascus spp. para la producción de
Tabla 1. Producción de pigmentos de Monascus en FMS

\begin{tabular}{|c|c|c|}
\hline Especie & Residuo agroindustrial & Referencia \\
\hline $\begin{array}{l}\text { M. purpureus } \\
\text { LPB97 }\end{array}$ & $\begin{array}{l}\text { Semilla de sandía, } \\
\text { salvado de arroz, } \\
\text { salvado de trigo, } \\
\text { residuos de la industria } \\
\text { de aceite de coco, de } \\
\text { sésamo y de aceite de } \\
\text { cacahuate, polvo de } \\
\text { yuca y polvo de semillas } \\
\text { de tamarindo }\end{array}$ & $\begin{array}{l}\text { Babitha et } \\
\text { al. (2007) }\end{array}$ \\
\hline M. ruber $102 \mathrm{w}$ & $\begin{array}{c}\text { Granos de arroz en } \\
\text { polvo }\end{array}$ & $\begin{array}{l}\text { Lian et al. } \\
\text { (2007) }\end{array}$ \\
\hline $\begin{array}{l}\text { M. ruber } \\
\text { MTCC2326 }\end{array}$ & Arroz triturado & $\begin{array}{l}\text { Vidyalakshmi } \\
\text { et al. (2009) }\end{array}$ \\
\hline $\begin{array}{l}\text { M.purpureus } \\
\text { KACC42430 }\end{array}$ & Olote de maíz & $\begin{array}{l}\text { Velmurugan } \\
\text { et al. (2011) }\end{array}$ \\
\hline $\begin{array}{l}\text { M. purpureus } \\
\text { CECT2955 }\end{array}$ & $\begin{array}{c}\text { Cáscaras de naranja, } \\
\text { mandarina, toronja, } \\
\text { limón, piña, tuna, } \\
\text { mango, papaya, Aloe } \\
\text { vera, algas marinas y } \\
\text { vaina de frijol }\end{array}$ & $\begin{array}{c}\text { Velázquez } \\
\text { Arellano } \\
\text { (2013) }\end{array}$ \\
\hline
\end{tabular}

Elaboración propia.

pigmentos han reportado una temperatura óptima de $30{ }^{\circ} \mathrm{C}$ (Babitha et al., 2007; Lian et al., 2007; Velmurugan et al., 2011; Priatni et al., 2014). Por otro lado, Carvalho et al. (2006) obtuvieron la mayor producción de pigmento en FMS por Monascus spp. a una temperatura de $32^{\circ} \mathrm{C}$.

Por otro lado, el pH del sustrato, al igual que la temperatura del sistema, es uno de los factores más importantes para determinar el crecimiento microbiano y la actividad metabólica en FMS. El rendimiento de producción de tintes por Monascus ha sido estudiado por algunos autores empleando diferentes niveles iniciales de $\mathrm{pH}$. Yongsmith et al. 
(2000) reportó que la mayor producción de pigmentos amarillos por Monascus se encontró en niveles de $\mathrm{pH}$ entre 5.0 y 6.0, mientras que la máxima producción de tintes rojos se observó a valores de $\mathrm{pH}$ de 5.0. Por otro lado, se ha reportado que a valores de rango de $\mathrm{pH}$ ácido (1.0 2.0) y neutro-básico (7.0-8.0) la producción de pigmentos disminuye (Velmurugan et al., 2011).

Humedad relativa. La humedad relativa en procesos de FMS es un parámetro clave para controlar el crecimiento de microorganismos y biosíntesis de los metabolitos de interés (Pandey, A., 2003). Se ha reportado que cada cepa de Monascus necesita su propio contenido de humedad inicial en el sustrato, óptima para la producción de pigmento (Lotong y Suwanarit, 1990). Diversos estudios indican que un contenido de humedad en el sustrato menor a $40 \%$ $(\mathrm{v} / \mathrm{p})$ conduce a menor producción de pigmento, se mostró la mayor producción para humedades mayores a 50\% (v/p) (Johns y Stuart, 1991; Babitha et al., 2007). En este sentido, Velmurugan et al. (2011) reportaron la mayor producción de pigmentos (amarillo y rojo de $18.92 \mathrm{DO} 412 \mathrm{~nm}$ y 14.26 DO500nm, respectivamente) con un nivel de humedad de 60 \%. De igual forma, Yongsmith et al. (2000) obtuvieron la mayor producción de pigmento con humedades de $60 \%$ con una cepa de Monascus spp. Cabe destacar que ambos estudios concordaron en que la baja productividad registrada en los sistemas en que la humedad relativa fue superior a $65 \%$ se debió a la aglomeración del sustrato causada por la formación de lodos disminuyendo la oxigenación en el medio y, por tanto, inhibiendo el crecimiento del microorganismo. En contraste, Srianta y Harijono (2015) y Said et al. (2010) reportaron el uso de una humedad óptima de $70 \%$ para la producción de pigmentos por Monascus purpureus y Monascus ruber, respectivamente, en sistemas empacados, lo que muestra que el empleo de un sistema aireado puede mantener condiciones de humedad alta favoreciendo la de oxigenación del microorganismo, lo que evita la formación de lodos, mejora la transferencia de masa y permite el crecimiento y producción de colorantes por parte del microorganismo.

Tamaño de partícula. El tamaño de partícula es uno de los parámetros relacionados con la transferencia de masa entre el microorganismo-sustrato en FMS (Díaz Sánchez, 2009). En tamaños de partícula pequeños el área de contacto entre el micelio del microorganismo y sustrato se incrementa, a su vez limita el intercambio de gases al reducirse el espacio entre partículas. Por el contrario, a mayor tamaño de partícula el área de contacto se reduce e incrementa el intercambio de gases al generarse mayor espacio entre las partículas del sustrato (Srianta y Harijono, 2015). Estos dos efectos opuestos pueden influir en los niveles de producción de metabolitos de interés, porlo que es necesario seleccionar el tamaño de partícula adecuado para la producción del metabolito de interés (Botella et al., 2007). Babitha et al. (2006) evaluó la producción de colorantes por Monascus en FMS empleando semillas de la fruta jaca y observó que la máxima producción de pigmentos se obtuvó empleando tamaños de partícula entre 0.4 y $0.6 \mathrm{~mm}$. En contraste, Velmurugan et al. (2011) obtuvieron el mayor rendimiento del tinte por Monascus en FMS con olote de maíz a un tamaño de partícula 2.0 $\mathrm{mm}$. Es importante observar los diferentes rangos de tamaño de partícula empleados, por lo que es recomendable que para cada sustrato a emplear en FMS se lleve a cabo un estudio preliminar del impacto del tamaño de partícula del sustrato sobre el rendimiento del proceso.

Aireación. La aireación en las fermentaciones en medio sólido es más fácil de controlar que en fermentaciones sumergidas, porque la superficie de contacto es mayor entre el aire y el líquido que está absorbido en las partículas (Viniegra González et al., 2003). Said et al. (2010) reportaron que para una fermentación sólida en camas empaquetadas con Monascus ruber, una velocidad de aireación forzada mayor a $0.5 \mathrm{l} / \mathrm{min}$ redujo la producción de pigmentos y biomasa como consecuencia de la pérdida de agua, mientras que los niveles más altos de colorantes se obtuvieron a tasas de aireación forzada de entre 0.05 y $0.2 \mathrm{l} / \mathrm{min}$, en donde se alcanzó un contenido de pigmentos de $98.0 \mathrm{DO} / \mathrm{g}$ de materia seca en la cama.

\section{Residuos agroindustriales}

Los residuos generados tienen alta demanda biológica de oxígeno y pueden causar problemas de diferentes aspectos, incluso costos de recolección, eliminación, tratamiento y pérdida de materias primas valiosas (Kasinski y Wojnowska Baryla, 2014). El interés científico sobre el cuidado del medio ambiente con disminuir los niveles de contaminación se está viendo favorable con la recuperación de una extensa gama de subproductos industriales (Saval, 2012). En el caso particular de los pigmentos microbianos, que presentan grandes ventajas en términos de producción en comparación con 


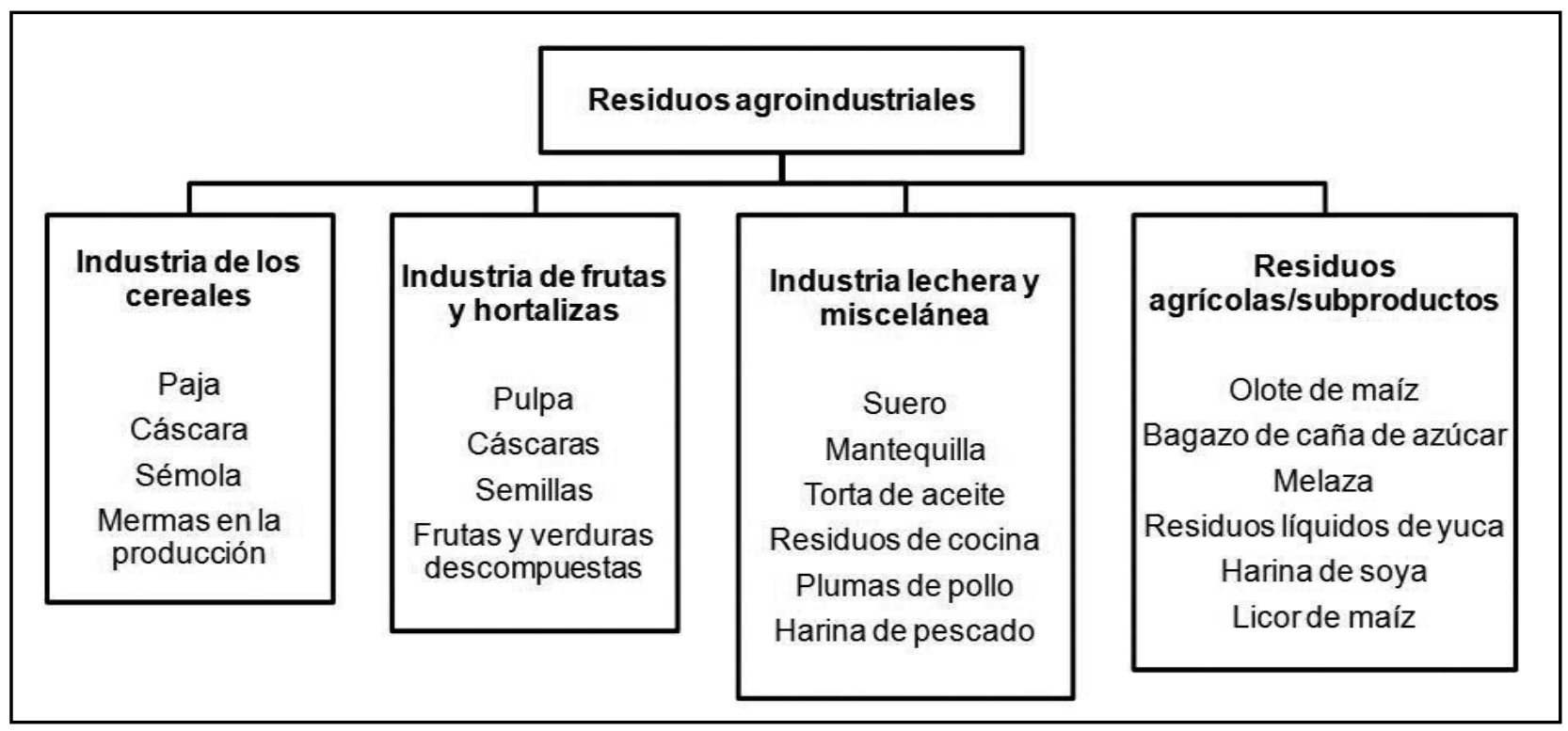

Figura 2. Tipos de residuos agroindustriales generados por diferentes industrias comúnmente empleados en procesos biotecnológicos (Panesar et al., 2015).

tintes similares extraídos de vegetales o animales, estrategias de producción empleando bioprocesos que utilicen residuos agroindustriales serán de gran importancia en desarrollo de la industria alimentaria, por mencionar algunas. Actualmente, diversos residuos derivados de agroindustrias ya están siendo utilizados para producción de pigmentos naturales. En la Figura 2 se muestran los residuos agroindustriales que han sido empleados en procesos biotecnológicos para la obtención de pigmentos, enzimas y otros metabolitos de interés comercial (Panesaretal., 2015).

Actualmente, en el norte de México se generan gran cantidad de residuos agroindustriales que pueden ser aprovechados en la producción de pigmentos vía FMS. Dentro de los principales productos que se encuentran en la región semidesértica y con potencial se pueden mencionar residuos del proceso de extracción de cera de candelilla, residuos del proceso de extracción de fibra de lechuguilla (Agave lechuguilla), orégano (Origanum vulgare L.), maguey (Agave americana L.), jojoba (Simmonsdia chinensis), sábila (Aloe vera), nopal (Opuntia spp.) y damiana (Turnera afrodisiaca), entre otros. Por tanto, con base en el potencial de los resiudos antes mencionados, se abre un área de oportunidad para el desarrollo de bioprocesos para la bioproducción de pigmentos en FMS.

\section{CONCLUSIONES}

La producción de pigmentos naturales de origen fúngico como los de las cepas de Monascus representan una fuente prometedora y sustentable para cubrir las necesidades comerciales de estos compuestos de interés. Además, la producción de estos metabolitos posee la ventaja de su biosintetización mediante fermentación sólida, lo que abre la posibilidad de emplear residuos agroindustriales de fácil acceso con base en las industrias establecidas en la región.

\section{Agradecimientos}

La autora agradece al Consejo Nacional de Ciencia y Tecnología (Conacyt) por el apoyo financiero brindado para la realización de estudios de Maestría (559147). 
LITERATURA CITADA

- BABITHA, S. et al. Jackfruit seed-A novel substrate for the production of Monascus pigments through solid-state fermentation. Food Technology and Biotechnology, 44, 465471, 2006.

- BABITHA, S. et al. Solid-state fermentation for the production of Monascus pigments from jackfruit seed. Bioresource Technology, 98(8): 1554-1560, 2007.

- BECHTOLD, T. et al. Extraction of natural dyes for textile dyeing from coloured plant wastes released from the food and beverage industry. Journal of the Science of Food and Agriculture, 86(2): 233-242, 2006.

- BENAVENTE VALDÉS, J. R. et al. Strategies to enhance the production of photosynthetic pigments and lipids in chlorophycae species. Biotechnology Reports, 10, 117-125, 2016.

- BOtella, $C$. et al. Xylanase and pectinase production by Aspergillus awamori on grape pomace in solid state fermentation. Process Biochemistry, 42 (1): 98-101, 2007.

- CARVAlho, J. C. et al. Production of Monascus biopigments: an overview. Agro FOOD industry hi-tech, 14(6): 37-42, 2003.

- CARVALHO, J. C. et al. Relation between growth, respirometric analysis and biopigments production from Monascus by solidstate fermentation. Biochemical Engineering Journal, 29(3): 262-269, 2006.

- CelestinO, J. D. R. et al. Bioprospecting of Amazon soil fungi with the potential for pigment production. Process Biochemistry, 49(4): 569-575, 2014.

- CHENG, M. J. et al. Secondary metabolites produced by the fungus Monascus pilosus and their anti-inflammatory activity. Phytochemistry Letters, 5(3): 567-571, 2012.

- DÍAZ SÁNCHEZ, A. B. Reciclado del orujo de uva como medio sólido de fermentación para la producción de enzimas hidrolíticas de interés industrial. Tesis doctoral. España: Universidad de Cádiz, 2009.

- DUFOSSÉ, L. et al. Microorganisms and microalgae as sources of pigments for food use: a scientific oddity or an industrial reality? Trends in Food Science \& Technology, 16(9): 389-406, 2005.

- DURSUN, D. y DALGIÇ, A. C. Optimization of astaxanthin pigment bioprocessing by four different yeast species using wheat wastes. Biocatalysis and Agricultural Biotechnology, 7 , $1-6,2016$.

- GENERAL, T. et al. Fungal utilization of a known and safe macroalga for pigment production using solid-state fermentation. Journal of Applied Phycology, 26(3): 1547-1555, 2014.

- HAN, O. y MUDGETT, R. E. Effects of oxygen and carbon dioxide partial pressures on Monascus growth and pigment production in solid-state fermentation. Biotechnology Progress, 8, 5-10, 1992.

- HSU, W. H. y PAN, T. M. Monascus purpureus-fermented products and oral cancer: a review. Applied Microbiology and Biotechnology, 93(5): 1831-1842, 2012.

- JOHNS, M. R. y STUART, D. M. Production of pigments by Monascus purpureus in solid culture. Journal of Industrial Microbiology, 8(1): 23-28, 1991.

- KASINSKI, S. y WOJNOWSKA BARYLA, I. Oxygen demand for the stabilization of the organic fraction of municipal solid waste in passively aerated bioreactors. Waste management, 34 (2): 316-322, 2014.

- $\quad$ LI, C. H. et al. Safety and mutagenecity evaluation of red mold dioscorea fermented from Monascus purpureus NTU 568. Food and Chemical Toxicology. 67, 161-168, 2014.

- LIAN, X. et al. Identification of new red pigments produced by Monascus ruber. Dyes and Pigments, 73(1): 121-125, 2007.

- LOTONG, N. y SUWANARIT, P. Fermentation of ang-kak in plastic bags and regulation of pigmentation by initial moisture content. Journal of Applied Microbiology, 68(6): 565-570, 1990.

- MORALES OYERVIDES, L. et al. Effect of heat exposure on the colour intensity of red pigments produced by Penicillium purpurogenum GH2. Journal of Food Engineering, 164, 21-29, 2015.

- NAM, K. et al. Antiobesity effect of a jelly food containing the L-tryptophan derivative of Monascus pigment in mice. Journal of Functional Foods, 9, 306-314, 2014.

- PANDEY, A. Solid-state fermentation. Biochemical Engineering Journal, 13(2-3): 81-84, 2003.

- PANDEY, A. et al. New developments in solid-state fermentation: I-bioprocesses and products. Process Biochemistry, 35(10): 1153-1169, 2000.

- PANDEY, S. et al. Coping with drought in rice farming in Asia: insights from a cross-country comparative study. Agricultural Economics, 37(s1): 213-224, 2007.

- $\quad$ PANESAR, R. et al. Production of microbial pigments utilizing agro-industrial waste: a review. Current Opinion in Food Science, 1, 70-76, 2015. 
- PONGRAWEe, N. y LUMYONG, S. Improving solid-state fermentation of Monascus purpureus on agricultural products for pigment production. Food and Bioprocess Technology, 4(8): 1384-1390, 2011.

- $\quad$ PRIATNI, S. et al. The utilization of solid substrates on Monascus fermentation for anticholesterol agent production. Procedia Chemistry, 9, 34-39, 2014.

- RAHARDJO, Y. S. P. et al. Modeling conversion and transport phenomena in solid-state fermentation: a review and perspectives. Biotechnology Advances, 24(2): 161-179, 2006.

- ROBLEDO, A. et al. Ellagic acid production by Aspergillus niger in solid state fermentation of pomegranate residues. Journal of Industrial Microbiology and Biotechnology, 35(6): 507-513, 2008

- RODRÍGUEZ COUTO, S. Exploitation of biological wastes for the production of value-added products under solid-state fermentation conditions. Biotechnology Journal, 3(7): 859-870, 2008.

- SAID, F. M. et al. The effects of forced aeration and initial moisture level on red pigment and biomass production by Monascus ruber in packed bed solid state fermentation. International Journal of Environmental Science and Development, 1 (1): 1-4, 2010.

- SAVAL, S. Aprovechamiento de Residuos agroindustriales: pasado, presente y futuro. BioTecnología, 16(2): 14-46, 2012.

- SRIANTA, I. y HARIJONO, A. Monascus-fermented sorghum: pigments and monacolin $K$ produced by Monascus purpureus on whole grain, dehulled grain and bran substrates. International Food Research Journal, 22(1): 377-382, 2015.

- STREIT, N. et al. Producción de pigmentos naturales (clorofila-a) en biorrefinerías agroindustriales. Ciencia y Tecnología, 8(2): 29-36, 2015.
- VELÁZQUEZ ARELLANO, M. E.Producción de pigmentos fúngicos (Monascus purpureus 2955) en residuos agroindustriales por fermentación sólida. Tesis de licenciatura. Coahuila, México: Universidad Autónoma Agraria Antonio Narro, 2013.

- Velmurugan, P. et al. Monascus pigment production by solid-state fermentation with corn cob substrate. Journal of Bioscience and Bioengineering, $112(6)$ : 590-594, 2011.

- VIDYALAKSHMI, R et al. Microbial bioconversion of rice broken to food grade pigments. Global Journal of Biotechnology \& Biochemistry, 4 (2): 84-87, 2009.

- VINIEGRA GONZÁLEZ, G. et al. Advantages of fungal enzyme production in solid state over liquid fermentation systems. Biochemical Engineering Journal, 13, 157-167, 2003.

- WANG, B. et al. Investigation of relationship between lipid and Monascus pigment accumulation by extractive fermentation. Journal of Biotechnology, 212, 167-173, 2015.

- WANI, K. S. et al. Pigment production. In Concise encyclopedia of Bioresource Technology, 2, 645-652, 2004.

- YANG, J. et al. Effect of oxygen supply on Monascus pigments and citrinin production in submerged fermentation. Journal of Bioscience and Bioengineering, 119(5): 564-569, 2015.

- YONGSMITH, B. et al. Color mutants of Monascus sp. KB9 and their comparative glucoamylases on rice solid culture. Journal of Molecular Catalysis. B, Enzymatic, 10(1-3): 263-272, 2000.

- ZANG, C. Z. et al. Identification and enhanced production of prodigiosin isoform pigment from Serratia marcescens N10612. Journal of the Taiwan Institute of Chemical Engineers, 45, 1133 1139,2014 